\title{
ON THE VIBRATIONS OF A CENTRIFUGE WITH THE BASKET IN CONSOLE
}

\author{
BOIANGIU, M[ihail] \& BOIANGIU, A[dina]
}

\begin{abstract}
This paper approaches a field of advanced research: the study of vibrations of industrial centrifuges by applying the transfer matrix method. It will be established a study model considering the shaft as continuous medium. Bending vibrations coupled with axial vibrations will be studied. The transfer matrix and the transition matrices will be established. The case of the free vibrations will be developed. The values for the natural frequencies will be compared with them obtained by neglecting the coupling of vibrations.
\end{abstract}

Keywords: ccentrifuge, transfer matrix method, vibrations, natural frequencies, coupled vibrations.

\section{INTRODUCTION}

The centrifuge is a mechanical system composed of a flexible shaft and a basket mounted on the shaft (Fig. 1). The weight of the basket is much bigger than that of the shaft. This machine realizes the process named centrifugal action. This is a mechanical process, which allows the acceleration separation of the components of heterogeneous systems.

The shaft that is quiet in bearing can be horizontal positioned or upright. In this paper the horizontal centrifuge is considered [1].

Anyway the reserved chapter of the dynamic behaviour of these machines is small, and the problem is treatise through very simplify methods. In some books there is not approach about centrifuge vibration [2]. In the field of activities are only few paper works, or this aim is not about the dynamics centrifuge machine. Some paper works treat the approximate and construction of the subassembly [3] and other refers to the separation process of blending stock [4], [5].

This study has as objective the improvement of the study of vibrations of the centrifuge with the basket in console. That is why the study model is flexible shaft considered to be a continuous medium positioned on two bearing blocks and a basket fixed on the shaft. Also the study takes account of the coupling between the bending vibrations and the axial vibrations.

By knowing the natural frequencies, and also the critical speed, it will be avoided the operation of the centrifuge at this speed or around this kind of speed [6].

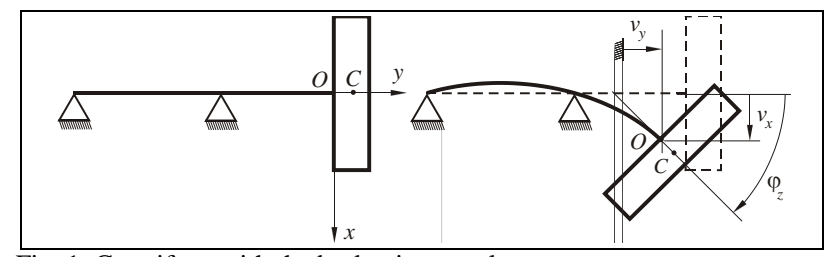

Fig. 1. Centrifuge with the basket in console

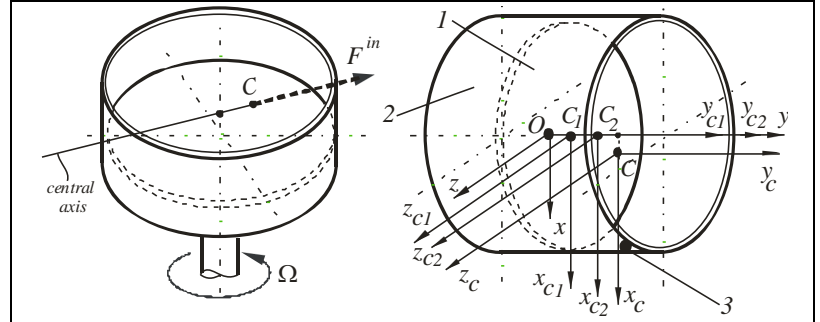

Fig. 2. The basket

\section{THE BASKET}

Each centrifuge has a basket. It is considered that the basket is fixed on the shaft in the point $\mathrm{O}$ (Fig. 2) [3].

In the case of the centrifuges, the basket cannot be considered a thin disk and the fixing point of the basket cannot be in the centre of mass of the basket.

The centre of mass of the basket (including the operating material) is not placed on the revolution axis of the centrifuge. The reasons are the shape of the basket and the operational features. The shape of the basket and the uneven deposits of the operating material on the wall of the basket are the reasons for which the axes $O x, O z$ and $O y$ are not axes of symmetry and the centre of mass of the basket is not on the $O y$ axis.

The d'Alembert's principle is applied [7]. The revolution speed of the centrifuge is constant. The line of action of the resultant of d'Alembert's fictitious forces of the basket (operating material included) crosses the revolution axis. The resultant moment of d'Alembert's fictitious forces is calculated with respect to the fixing point of the basket, $\mathrm{O}$.

Using the notations:

$\vec{F}^{\text {in }}$ for the resultant of d'Alembert's fictitious forces;

$m$ for the mass of the basket;

- $\quad \xi, \eta, \zeta$ for the coordinates of the centre of mass;

- $v_{x}$ for the displacement for bending vibration

$v_{x}=V_{x}(y) \cos (\omega t-\phi)$;

- $\quad v_{y}$ for the displacement for axial vibration

$v_{y}=V_{y}(y) \cos (\omega t-\phi)$;

- $\varphi_{z}$ for the rotation of the cross section

$$
\begin{gathered}
\varphi_{z}=\frac{d v_{x}}{d y}=\Phi_{z}(y) \cos (\omega t-\phi), \\
\omega_{z}=\frac{d \varphi_{z}}{d t}=-\omega \Phi_{z}(y) \sin (\omega t-\phi), \\
\varepsilon_{z}=\frac{d^{2} \varphi_{z}}{d t^{2}}=-\omega^{2} \varphi=-\omega^{2} \frac{d v_{x}}{d y} ;
\end{gathered}
$$


- $\omega_{z}$ for the angular speed;

- $\varepsilon_{z}$ for the angular acceleration;

- $\vec{M}_{O}^{i n}$ for the resultant moment of d'Alembert's fictitious forces with respect to the point $\mathrm{O}$;

- $J_{c z}$ for the moment of inertia with respect to $\mathrm{Cz}$ axis;

- $J_{c x z}, J_{c y z}$ are products of inertia;

- $\omega$ for the natural angular frequency of $n$ order;

it is obtained for the resultant and the moment resultant of d'Alembert's fictitious forces:

$$
\left\{\begin{array}{l}
F_{x}^{i n}=m\left(\omega^{2} v_{x}+\varepsilon_{z} \eta+\omega_{z}^{2} \xi\right) \\
F_{y}^{i n}=m\left(\omega^{2} v_{y}-\varepsilon_{z} \xi+\omega_{z}^{2} \eta\right) \\
F_{z}^{i n}=0
\end{array}\right.
$$

$\left\{\begin{array}{l}M_{o x}^{i n}=\left(J_{c x z}+m \xi \zeta\right) \varepsilon_{z}-\left(J_{c y z}+m \eta \zeta\right) \omega_{z}^{2} \\ M_{o y}^{i n}=\left(J_{c y z}+m \eta \zeta\right) \varepsilon_{z}+\left(J_{c x z}+m \xi \zeta\right) \omega_{z}^{2}+m \zeta \omega^{2} v_{x} \\ M_{o z}^{i n}=\left(J_{c z} \omega^{2}+m \omega^{2} \xi^{2}+m \omega^{2} \eta^{2}\right) \varphi_{z}-m \eta \omega^{2} v_{x}+m \xi \omega^{2} v_{y}\end{array}\right.$

It is considered that the centers of mass of the bottom and wall of the basket are situated on the revolution axis of the centrifuge. In these conditions the uneven deposits of the operating material on the wall of the basket is the reason for which the centre of mass of the basket is not placed on the revolution axis of the centrifuge. The bending vibrations in the plane that includes the centre of mass of the basket and the revolution axis will be studied $(\zeta=0)$ (Fig. 1).

Also, because of the high rigidity, the amplitude of the rotation of the cross section $\varphi_{z}$ has low values. $\omega_{z}^{2}$ has been neglected with respect $\varepsilon_{z}$. In these conditions the expressions of the resultant and the moment resultant of d'Alembert's fictitious forces can be written:

$$
\left\{\begin{array}{l}
F_{x}^{i n}=m \omega^{2} v_{x}-m \eta \omega^{2} \varphi_{z} \\
F_{y}^{i n}=m \omega^{2} v_{y}+m \xi \omega^{2} \varphi_{z} \\
F_{z}^{i n}=0
\end{array}\right.
$$

$\left\{\begin{array}{l}M_{o x}^{i n}=0 \\ M_{o y}^{i n}=0 \\ M_{o z}^{i n}=\left(J_{c z} \omega^{2}+m \xi^{2} \omega^{2}+m \eta^{2} \omega^{2}\right) \varphi_{z}-m \eta \omega^{2} v_{x}+m \xi \omega^{2} v_{y}\end{array}\right.$

From the expressions of the resultant and the moment resultant of d'Alembert's fictitious forces it results that the axial vibrations and the bending vibrations are coupled.

\section{DESCRIPTION OF METHOD}

\subsection{Hypothesis}

Keeping account of (2), it is considered that the shaft consists of straight elements of constant section which effect bending vibrations in a plane which contain the center of mass of the basket and the longitudinal axis of the shaft and axial vibrations along this axis (Fig. 1).

It is considered that all the elements are compact and homogeneous. It is neglected the dimensions of the bearings.

It was constructed [8] a transfer matrix which keeps account of these types of vibrations.

\subsection{Transfer matrix for one element of the shaft}

The matrix relation between the state vectors of ends of an element $\mathrm{k},\{q\}_{k-1}^{r}$ and $\{q\}_{k}^{l}$ respectively, can be written:

$$
\left\{\begin{array}{c}
v_{x} \\
-\frac{\varphi_{z}}{\alpha_{i}} \\
-\frac{M_{z}}{\alpha_{i}^{2} E I} \\
-\frac{F_{x}}{\alpha_{i}^{3} E I} \\
v_{y} \\
\frac{F_{y}}{\alpha_{c} E A}
\end{array}\right\}_{k}^{l}=\left[\begin{array}{cccccc}
S & T & U & V & 0 & 0 \\
V & S & T & U & 0 & 0 \\
U & V & S & T & 0 & 0 \\
T & U & V & S & 0 & 0 \\
0 & 0 & 0 & 0 & M & N \\
0 & 0 & 0 & 0 & -N & M
\end{array}\right]_{k}\left\{\begin{array}{c}
v_{x} \\
-\frac{\varphi_{z}}{\alpha_{i}} \\
-\frac{M_{z}}{\alpha_{i}^{2} E I} \\
-\frac{F_{x}}{\alpha_{i}^{3} E I} \\
v_{y} \\
\frac{F_{y}}{\alpha_{c} E A}
\end{array}\right\}_{k-1}^{r}
$$

where:

- $M_{z}$ bending moment;

- $F_{x}$ shearing force;

- $\quad F_{y}$ axial force;

- S, T, U, V - Krilov - Rayleigh functions:

$S=\frac{1}{2}[\cosh (\alpha l)+\cos (\alpha l)], T=\frac{1}{2}[\sinh (\alpha l)+\sin (\alpha l)]$,

$U=\frac{1}{2}[\cosh (\alpha l)-\cos (\alpha l)], \quad V=\frac{1}{2}[\sinh (\alpha l)-\sin (\alpha l)] ;$

- $\mathrm{M}, \mathrm{N}$ - symbols for abbreviation:

$$
M=\cos \alpha_{c} l, \quad N=\sin \alpha_{c} l
$$

- $\quad l$ the length of an element;

- $\quad \alpha_{i}=\sqrt{\omega} \sqrt[4]{\frac{\rho A}{E I}}$

- $\quad \alpha_{c}=\omega \sqrt{\frac{\rho}{E}}$;

- $\rho$ density of material;

- $A$ area of cross section;

- E Young's modulus (longitudinal modulus of elasticity);

- $\quad I$ - second moment of area.

The matrix $(6 \times 6)$, named $[A]_{k}$, is the transfer matrix for an element of the shaft.

\subsection{Special matrices}

In some nodes it is possible to appear special matrices. Between the left state vectors and right state vectors of the section $\mathrm{k},\{q\}_{k}^{l}$ and $\{q\}_{k}^{r}$ respectively, we can write relation:

$$
\{q\}_{k}^{r}=[S]_{k}\{q\}_{k}^{l},
$$

The square matrix $(6 \times 6)[S]_{k}$ can be one from the matrices:

- saltus matrix, $[\mathrm{B}]_{\mathrm{k}}$, when in a node $\mathrm{k}$ appears a saltus of section. The non-zero elements of this matrix are:

$$
\begin{aligned}
& B_{1,1}=B_{5,5}=1 ; B_{2,2}=\frac{a_{k}}{b_{k}} ; B_{3,3}=a_{k}^{2} b_{k}^{2} ; B_{4,4}=a_{k}^{3} b_{k} \\
& B_{6,6}=a_{k}^{4} ; \text { where: } a_{k}=\sqrt[4]{\frac{A_{k}}{A_{k+1}}} ; b_{k}=\sqrt[4]{\frac{I_{k}}{I_{k+1}}} .
\end{aligned}
$$

- transition matrix, $[\mathrm{E}]_{\mathrm{k}}$, over an elastic bearing in a node $\mathrm{k}$ (Fig. 3). The non-zero elements of this matrix are:

$$
\begin{gathered}
E_{1,1}=E_{2,2}=E_{3,3}=E_{4,4}=E_{5,5}=E_{6,6}=1 ; \\
E_{4,1}=-\frac{K_{x}}{\alpha_{i}^{3} E I} ; E_{6,5}=\frac{K_{y}}{\alpha_{c} E A} .
\end{gathered}
$$




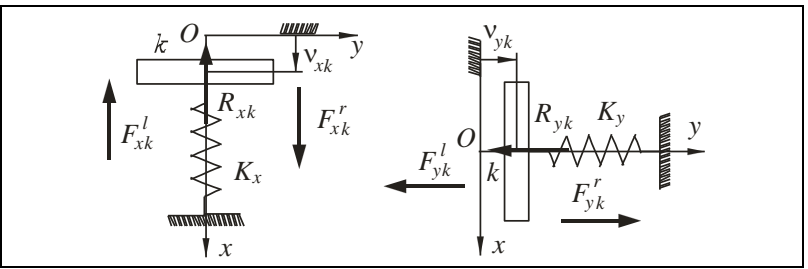

Fig. 3. Elastic bearing

$\mathrm{K}_{\mathrm{x}}$ and $\mathrm{K}_{\mathrm{y}}$ are the bearing's elastic constants on the directions $\mathrm{Ox}$ and $\mathrm{Oy}$ respectively. If the elastic bearing is radial the element $E_{6,5}$ is zero. If the elastic bearing is radial-axial the element $E_{6,5}$ is non-zero.

- transition matrix, $[\mathrm{D}]_{\mathrm{k}}$, over the basket fixed in a node $\mathrm{k}$. The non-zero elements of this matrix are:

$$
\begin{gathered}
D_{1,1}=D_{2,2}=D_{3,3}=D_{4,4}=D_{5,5}=D_{6,6}=1 \\
D_{4,1}=\frac{m \alpha_{i}}{\rho A} ; D_{4,2}=\frac{m \eta \alpha_{i}^{2}}{\rho A} ; D_{6,2}=\frac{m \xi \alpha_{i} \alpha_{c}}{\rho A} ; D_{6,5}=-\frac{m \alpha_{c}}{\rho A} ; \\
D_{3,1}=-\frac{m \eta \alpha_{i}^{2}}{\rho A} ; D_{3,2}=-\frac{\left(J_{c z}+m \xi^{2}+m \eta^{2}\right) \alpha_{i}^{3}}{\rho A} ; D_{3,5}=\frac{m \xi \alpha_{i}^{2}}{\rho A} .
\end{gathered}
$$

If it take into account the gyroscopic moment [8] (Fig. $4)$, only the projection of the gyroscopic moment on the axis $\mathrm{Oz}, M_{z}$, will be considered, because the bending vibrations in the plane $\mathrm{xOy}$ are studied in this paper.

It is obtained:

- for the forward precession

$$
M_{z}=\left(J_{c y} \frac{\Omega}{\omega}-J_{c z}-m \eta^{2}\right) \omega^{2} \varphi_{z}+m \eta \omega^{2} v_{x} ;
$$

- for the backward precession

$$
M_{z}=-\left(J_{c y} \frac{\Omega}{\omega}+J_{c z}+m \eta^{2}\right) \omega^{2} \varphi_{z}+m \eta \omega^{2} v_{x} .
$$

$\Omega$ is the revolution speed. In the transition matrix, $[\mathrm{D}]_{\mathrm{k}}$, only the element $D_{3,2}$ will be modified:

- for the forward precession

$$
D_{3,2}=-\left(-J_{c y} \frac{\Omega}{\omega}+J_{c z}+m \xi^{2}+m \eta^{2}\right) \frac{\alpha_{i}^{3}}{\rho A} .
$$

- for the backward precession

$$
D_{3,2}=-\left(J_{c y} \frac{\Omega}{\omega}+J_{c z}+m \xi^{2}+m \eta^{2}\right) \frac{\alpha_{i}^{3}}{\rho A} .
$$

\subsection{Transfer matrix for the shaft-basket system}

The shaft-basket system is divided in straight elements which have constant section and which are not acted by concentrated forces or moments. The elements obtained are numbered in order beginning with number 1 for the first element from the left side (Fig. 5). The system will be traversed from the left to the right on the mean fibre.

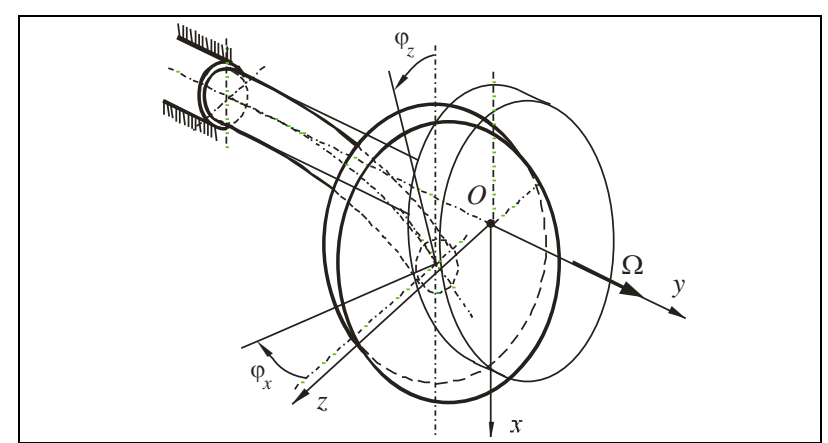

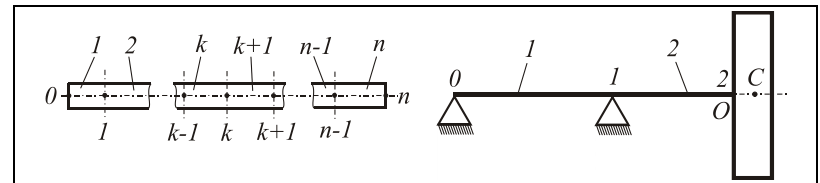

Fig. 5. Application of proposed method to a centrifuge

The points, where a saltus of section appears or act concentrated forces or moments, are named nodes. The numbering of the nodes begins with number 0 for the first node from the left side. So, the element number $k$ will be bounded by the nodes $k-1$, at the left side, and $k$ at the right side. For each element $k(k=1,2,---, n)$ is written the transfer matrix $[\mathrm{A}]_{\mathrm{k}}$.

In the state vectors for the nodes from the ends of the system, 0 and $n$, will appear the boundary conditions. In the internal nodes, $1,2,---, n-1$, intervene the matrices presented:

$[\mathrm{B}]_{\mathrm{k}}$ - saltus matrix;

$[D]_{k}$ - transition matrix over basket;

$[\mathrm{E}]_{\mathrm{k}}$ - transition matrix over an elastic bearing.

The matrix relations between the left and right state vectors of the nodes can be written. Replacing successively, it is obtained the matrix relation between the state vectors of ends from the $n$ element, $\{q\}_{n}^{l}$ and the first (point of start 0$)\{q\}_{0}^{r}$ :

$$
\{q\}_{n}^{l}=[Q]_{k}\{q\}_{0}^{r},
$$

where, is written

$$
[Q]=[A]_{n}[H]_{n-1} \ldots[A]_{k+1}[H]_{k}[A]_{k-1} \ldots[H]_{1}[A]_{1} .
$$

The square matrix $(6 \times 6)$, named [Q] is called transfer matrix for the shaft-basket system. $[\mathrm{H}]_{\mathrm{k}}$ can be one of the matrices $[\mathrm{B}]_{\mathrm{k},}[\mathrm{D}]_{\mathrm{k}},[\mathrm{E}]_{\mathrm{k}}$.

Depending of the type of the leaning we write the boundary conditions and we obtain an algebraic system. We put the condition that the system to admit non-zero solution and we obtain the natural angular frequencies.

\section{EXAMPLE}

The method presented is applied in a concrete case of a centrifuge (Fig. 5) [6]. It has been divided in 2 elements. It has been obtained 3 points. The centrifuge is elastically leaned. The bearing of the node 1 is radial. The bearing of the node 0 is radial-axial.

The geometrical and mechanical characteristics of the shaft-basket system are the following: length of the shaft (console part included): $0.8 \mathrm{~m}$; diameter of the shaft: 0.08 $\mathrm{m}$; diameter of the basket: $0.5 \mathrm{~m}$; length of the basket: $0.12 \mathrm{~m}$; distance from the bearing to the fixing point of the basket: $0.05 \mathrm{~m}$; density of the material of the shaft and basket: $7800 \mathrm{~kg} / \mathrm{m}^{3}$; longitudinal modulus of elasticity (Young's modulus): $21 \times 10^{10} \mathrm{~N} / \mathrm{m}^{2}$; revolution speed: 2000 r.p.m; bearing's elastic constants: $K_{x}=5 \times 10^{20}$ $\mathrm{N} / \mathrm{m}, K_{y}=5 \times 10^{20} \mathrm{~N} / \mathrm{m}$.

The mass of the basket (operating material included):

Fig. 4. Action of the gyroscopic moment 
$44.94 \mathrm{~kg}$; coordinates of the center of mass of the basket (operating material included): $\xi=0.003 \mathrm{~m}, \eta=0.0605 \mathrm{~m}$; mechanical moments of inertia: $J_{C y}=1.8755 \mathrm{~kg} \cdot \mathrm{m}^{2}$, $J_{C Z}=1.0628 \mathrm{~kg} \cdot \mathrm{m}^{2}$.

The first eight natural frequencies have been calculated. The values have been compared with them obtained by neglecting the axial vibrations.

The values are written in Tab. 1.

\begin{tabular}{|c|c|c|c|}
\hline \multirow[b]{2}{*}{$\begin{array}{c}\text { Mode } \\
\text { number }\end{array}$} & \multirow[b]{2}{*}{ precession } & \multicolumn{2}{|c|}{ Natural frequencies } \\
\hline & & $\begin{array}{c}\text { Bending } \\
\text { vibrations }(\mathrm{Hz})\end{array}$ & $\begin{array}{c}\text { Bending vibration } \\
\text { coupled with axial } \\
\text { vibrations }(\mathrm{Hz})\end{array}$ \\
\hline \multirow{3}{*}{ Mode 1} & backward & 118.33 & 118.33 \\
\hline & neglected & 133.50 & 133.49 \\
\hline & forward & 150.32 & 150.33 \\
\hline \multirow{3}{*}{ Mode 2} & backward & 393.19 & 393.19 \\
\hline & neglected & 396.75 & 396.75 \\
\hline & forward & 401.06 & 401.05 \\
\hline \multirow{3}{*}{ Mode 3} & backward & - & 746.15 \\
\hline & neglected & - & 746.16 \\
\hline & forward & - & 746.16 \\
\hline \multirow{3}{*}{ Mode 4} & backward & 1193.33 & 1193.31 \\
\hline & neglected & 1194.06 & 1194.06 \\
\hline & forward & 1194.84 & 1194.85 \\
\hline \multirow{3}{*}{ Mode 5} & backward & 2421.78 & 2421.78 \\
\hline & neglected & 2423.30 & 2423.37 \\
\hline & forward & 2424.91 & 2424.92 \\
\hline \multirow{3}{*}{ Mode 6} & backward & - & 3263.26 \\
\hline & neglected & - & 3263.26 \\
\hline & forward & - & 3263.26 \\
\hline \multirow{3}{*}{ Mode 7} & backward & 3470.30 & 3469.92 \\
\hline & neglected & 3475.60 & 3475.71 \\
\hline & forward & 3480.93 & 3480.96 \\
\hline \multirow{3}{*}{ Mode 8} & backward & 4685.87 & 4684.00 \\
\hline & neglected & 4689.35 & 4692.39 \\
\hline & forward & 4696.31 & 4696.31 \\
\hline
\end{tabular}

Tab. 1. The first eight natural frequencies

Using the study model in which the bending vibrations are coupled with the axial vibrations new natural frequencies are obtained: the natural frequencies numbers 3 and 6 . These values cannot be obtained when the coupling of vibrations is neglected.

It is confirmed that the backward precession decreases the values of the natural frequencies and the forward precession increases the values of the natural frequencies. Generally, the influence of the gyroscopic moment upon the natural frequencies is more when the bending vibrations are coupled with the axial vibrations. The influence of the gyroscopic moment upon the new values of the natural frequencies, obtained by the method presented in this paper, is quasi zero. Also, the influence of the gyroscopic moment upon the values of the natural frequencies is low for the higher order of the natural frequencies.

It is possible that the prevalent character of the new natural frequencies obtained in this paper to be axial vibrations.

\section{CONCLUSION}

The objective of this paper was to improve the study of vibrations of the centrifuges. The method presented, which takes account of the coupling of vibrations, can be considered better because it gives the possibility to obtain more natural frequencies.

The study has its limitations:

- The critical speeds are not calculated. Not all the natural frequencies give critical speeds in the operating domain.

- In this study the coupling of bending with axial vibrations had priority. So, the influence of other factors upon the natural frequencies has been neglected.

- The coupling with the torsional vibrations has been neglected.

The next steps of the research will are:

- The choosing of ordinary values for the bearing's elastic constants. In this paper the bearings have been considered practically rigid, because it has been chosen high values for the bearing's elastic constants.

- Study for discover the prevalent character (bending or axial) of the news modes of vibrations and, generally, for each mode of vibrations. How the coupling of vibrations changed the natural modes of vibrations.

- The influence of the constructive and operating specific features upon the natural frequencies and critical speeds can be also a subject for a next study.

The proposed method is flexible and can be easily applied on different types of centrifuges.

\section{REFERENCES}

[1] Jinescu, V. (1998). Utilaj tehnologic pentru industria de proces, Editura Tehnica, ISBN 978-973-31-2357-6, Bucharest

[2] Sutherland, K. \& Records, A. (2001). Decanter centrifuge, Handbook, ISBN 1-856173-69-0, Elsevier

[3] Iatan, R. et al. (1990). Calculul si constructia tamburelor centrifugelor; Stari de deformatii si de tensiuni in fundurile circulare plane, Revista de Chimie, No. 1, 1990, pp. 67, ISSN 0034-7752

[4] Haeberle, S.; Brenner, T.; Schlosser, H. P.; Zengerle, R. \& Ducree, J. (2005). Centrifugal Micromixery, Chemical Engineering \& Technology, Vol.28, Issue 5, May 2005, pp. 613-616, ISSN 09307516

[5] Stiborsky, M. \& Anlauf, H. (2003). Prediction of centrifugal Deliquoring for Changing Particle Composition, Chemical Engineering \& Technology, Vol. 26, Issue 5, May 2003, pp. 553557, ISSN 0930-7516

[6] Boiangiu, M. \& Boiangiu, A. (2010). Influence of the constructive and operational features on the centrifuges, In: $D A A A M$ International Scientific Book 2010, Chapter 40, Branko Katalinik, (Ed.), pp. 445-462, DAAAM International Vienna, ISBN 978-3901509-74-2, Vienna

[7] Radoi, M. \& Deciu, E. (1993). Mecanica, Editura Didactica si Pedagogica, ISBN 973-30-2917-3, Bucharest

[8] Boiangiu, M. \& Alecu, A. (1999). Coupled vibrations of crankshaft, Printech, ISBN 973-652-021-8, Bucharest 